\title{
Transmission de la Wuchereriose et de la Sétariose bovine :
}

\author{
Etude histologique de la traversée de la paroi stomacale \\ d'Anopheles gambiae A et d'Aedes aegypti par les microfilaires. (1)
}

\author{
par O. BAIN et J. BRENGUES
}

Laboratoire de Zoologie (Vers) associé au C.N.R.S. ( $\mathrm{P}^{r}$ A.-G. Chabaud), Muséum national d'Histoire naturelle, 57, rue Cuvier, F 75005 Paris et O.R.S.T.O.M. Centre Muraz de Bobo-Dioulasso, Haute-Volta

\section{Résumé}

L'étude histologique du passage des microfilaires dans l'hémocèle chez les 3 couples Filaires-vecteurs, $W$. bancrofti- $A$. gambiae A, $W$. bancrofti- $A$. aegypti et $S$. labiatopapillosa- $A$. aegypti confirme que les microfilaires traversent la paroi stomacale aux niveaux où les cellules digestives sont épaisses et que, bloquées par la lame basale résistante, elles séjournent dans les cellules épithéliales; icette phase intra-épithéliale des microfilaires semble être un vestige des localisations intracellulaires des ancêtres Spirurides.

Chez l'Anophèle, la facilitation (la proportion des microfilaires passant dans l'hémocèle augmente avec le nombre de microfilaires ingérées) semble s'expliquer par la formation d'excroissances de l'épithélium digestif provoquées par les microfilaires; ces hypertrophies constituent de nouveaux sites particulièrement favorables à la traversée par les microfilaires.

Chez l'Aedes, aucune image histologique n'explique la limitation (le nombre des microfilaires passant dans l'hémocèle ne dépasse pas un chiffre limite, quelle que soit l'importance de la prise de microfilaires).

(1) Ces recherches ont bénéficié du soutien financier de l'Organisation Mondiale de la Santé. 


\begin{abstract}
Summary
The study by histological techniques of the passage in the haemocoel of the microfilariae from 3 Filaria-vector couples $(W$. bancrofti- $A$. gambiae A, $W$. bancrofti-A. aegypti and $S$. labiatopapillosa-A. aegypti) is reported; confinmation is given of the fact that the microfilariae migrate through the stomach wall where the digestive cells are swollen and that, blocked by the resistant basal membrane, they sejourn in the epithelial cells. This intra-epithelial phasis seems to be a vestige of the intracellular stages of the spirurid ancestors.

In the Anopheles, the facilitation (the rate of the microfilariae reaching the haemocoel increases with the number of microfilariae ingested) can be explained by the formation of excrescences of the gut epithelium due to the passage of the microfilariae; these hypertrophies create new sites particularly favourable to the crossing of the microfilariae.

In the Aedes, no histological clue is found as to the cause of the limitation (the number of the microfilariae reaching the haemocoel do not exceed a limit number whichever is the number of microfilariae ingested).
\end{abstract}

\title{
Introduetion
}

Anopheles gambiae A est un des principaux vecteurs de la Filaire de Bancroft en Afrique Occidentale; une analyse numérique effectuée en Haute-Volta (Brengues et Bain, sous presse) montre qu'environ $30 \%$ des microfilaires ingérées par ce vecteur passent dans l'hémocèle et sont ainsi théoriquement capables d'évoluer en formes infestantes; elle révèle également que plus le repas est riche en microfilaires, plus le pourcentage de celles qui quittent l'estomac est élevé (phénomène de facilitation). Cette particularité est liée au vecteur et non à la microfilaire ; en effet, avec des Aedes aegypti gorgés dans les mêmes conditions que les Anophèles, on observe à l'inverse un phénomène de limitation : le nombre des microfilaires qui arrivent dans l'hémocèle ne dépasse pas un chiffre limite (inférieur à 20) quelle que soit la quantité de microfilaires ingérées. Un phénomène identique est mis en évidence chez le couple Setaria labiatopapillosa Aedes aegypti (Brengues et Bain, sous presse).

Nous essayons d'expliquer ces particularités par une étude histologique de ces 3 couples Filaires - vecteurs.

\section{I. - WUCHERERIA BANCROFTI - ANOPHELES GAMBIAE A}

Les microfilaires de $W$. bancrofti mesurent $280 \mu$ de long et $7 \mu$ de large ; elles se développent dạn les muscles thoraciques des Anophèles.

Le porteur de microfilaires est un Africain, originaire de Koupela, en Haute-Volta. 
Anopheles gambiae A est récolté à Tonogosso, en Haute-Volta. Les $q$ des générations $F_{1}$ et $F_{2}$ obtenues au laboratoire du Centre Muraz (Haute-Volta), et âgées de 3 à 6 jours, sont utilisées dans ces expériences. L'élevage est maintenu à $27^{\circ}$.

\section{Etude histologique de l'estomac d'A. gambiae A gorgé sur Homme sain. Son évolution pendant les premières 24 heures.}

Les Anopheles gambiae A témoins ont été gorgés sur Homme sain et fixés à $1 \mathrm{~h}$ $(2$ $), 3$ h (2 \$), 6 h (3 q) et 21 h ( 2 q). Après le repas, le cardia et l'estomac sont remplis de sang; le proctodeum est vide. Le repas de ces Moustiques est volumineux et il entraîne une grande distension de la paroi stomacale. L'épithélium digestif est donc très mince dans son ensemble (environ $2 \mu$ d'épaisseur). Mais il est plus épais en certaines zones : les cellules digestives sont toujours hautes $(15 \mu$ à $25 \mu)$ au voisinage du proctodeum ; les autres régions à épithélium assez épais ont une position variable et se présentent sous deux aspects : ce sont généralement des ensembles de cellules cubiques de $7 \mu$ à $15 \mu$ d'épaisseur formant des surfaces de $100 \mu$ à $150 \mu$ de côté, et parfois de petits groupes de cellules pincées à la base et épanouies en éventail dans la lumière stomacale, de $20 \mu$ à $30 \mu$ d'épaisseur (fig. 2, photo 1). Quand l'épithélium digestif est extrêmement distendu, par exemple sous la poussée des membranes intersegmentaires abdominales, il présente une coloration bleu vert (hémalun-éosine). Les noyaux des cellules stomacales ont une forme variable avec le degré d'étirement de l'épithélium ; ils sont très aplatis dans les zones minces; ils sont gros, ovoïdes, avec un nucléole et des grains de chromatine dans les zones épaisses. La bordure striée des cellules stomacales se discerne mal quand l'épithélium est étiré mais est nette dans la région préproctodéale et les surfaces épaisses. Au cours de la digestion (à partir de la $6^{\circ} \mathrm{h}$.), l'épithélium s'épaissit (fig. 1 A).

Le repas sanguin est rapidement (30' à 40') entouré par un coagulum difficile à délimiter ; il est généralement très mince $(1 \mu$ à $5 \mu)$, mais peut atteindre par place une vingtaine de $\mu$; il est mis en évidence par l'Azan et le Bleu Alcian (il se colore en bleu). Quelques lamelles apparaissent dans ce coagulum mais elles sont rares. Pendant les 12 premières heures qui suivent le repas, l'aspect du précurseur de la membrane péritrophique change peu; à la $18^{\circ}$ heure, le repas de sang est enfermé dans une croûte péritrophique grenue, également coloré par le Bleu Alcian ; elle est mince $(5 \mu$ à $7 \mu$ ) (fig. 1 A).

L'estomac est entouré par un réseau de fibres musculaires, particulièrement bien développées dans la région préproctodéale. A ce niveau la lame conjonctive qui relie ces fibres entre elles est bien visible.

\section{laires. \\ Etude histologique de la traversée de la paroi stomacale par les microfi-}

$32 \uparrow$ de Moustiques ont été coupées et observées: 2 à 15, $9 q$ à 30 , $7 q$ à 1 h, 6 à $3 \mathrm{~h}, 3$ à $6 \mathrm{~h}, 4 q$ à $12 \mathrm{~h}$ et $1 q$ à $21 \mathrm{~h}$. 

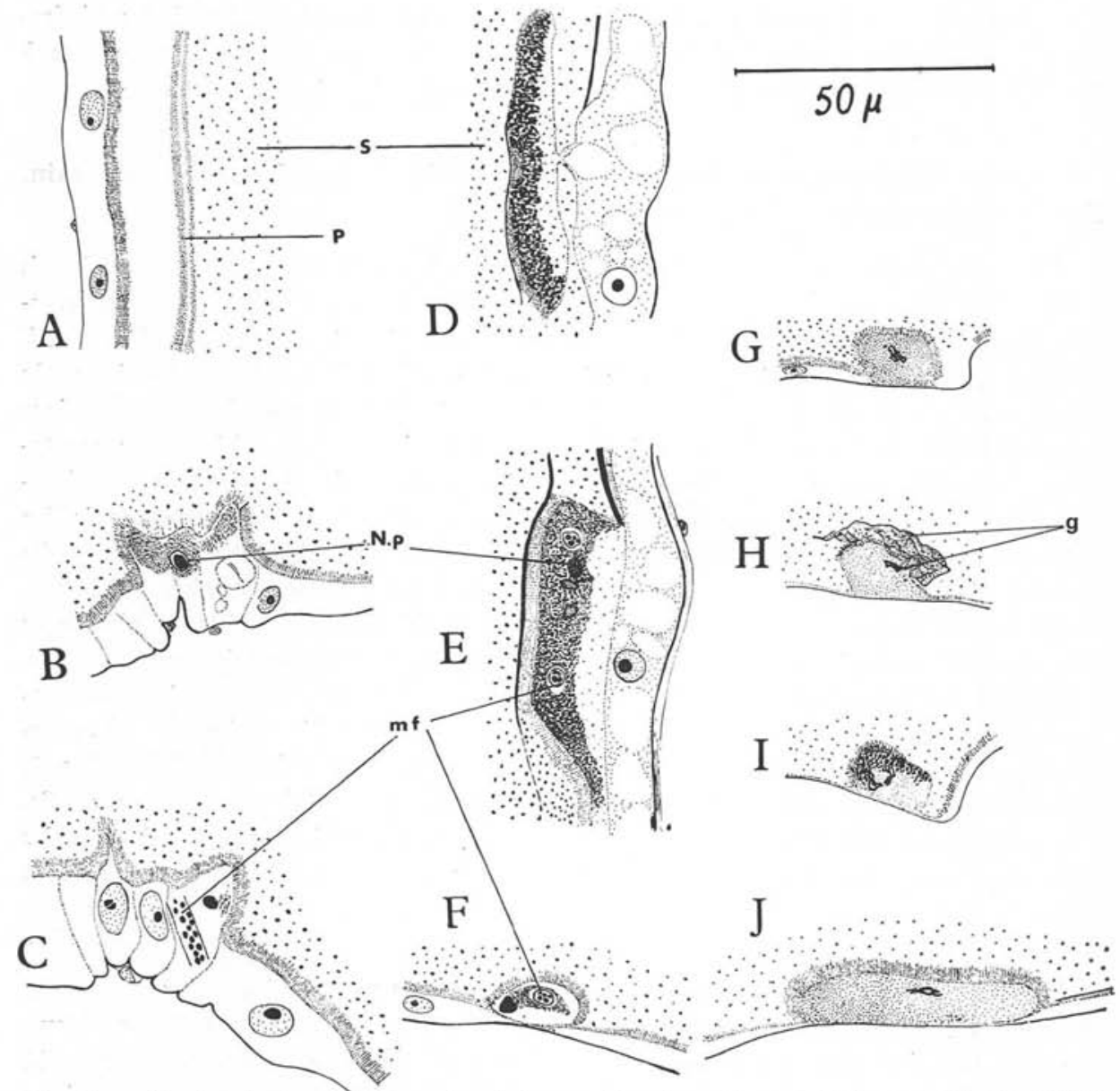

FIG. 1. - Wuchereria bancrofti chez Anopheles gambiae A. - A: aspect d'une membrane péritrophique, $24 \mathrm{~h}$ après le repas. - B et C: 2 coupes voisines de l'épithélium digestif, 30' après le repas, au niveau d'un groupe de cellules hautes; en B, vacuole laissée par le passage de la microfilaire, réaction du cytoplasme et apparition de noyaux picnotiques; en $\mathrm{C}$, section de la microfilaire dans une cellule digestive. - D et E: épithélium digestif avec formation « en parasol », $12 \mathrm{~h}$ après le repas; en E, 2 sections de microfilaires; le cytoplasme apical est fortement basophile. - F : épithélium digestif avec formation «en boule» contenant une microfilaire, $3 \mathrm{~h}$ après le repas. - G à I: épithélium digestif localement abrasé $(G)$ au voisinage d'une petite région hypertrophiée qui renferme partiellement une gaine de microfilaire $(3 \mathrm{~h}$ après le repas). - J : même phénomène, $6 \mathrm{~h}$ après le repas

\section{1) Répartition des microfilaires dans le repas de sang.}

Les microfilaires se répartissent dans toute la masse sanguine et ne se concentrent pas en des zones privilégiées. Elles traversent aisément le précurseur de la membrane péritrophique, mais à la $12^{\prime \prime} \mathrm{h}$ et à la $21^{\circ} \mathrm{h}$, de rares microfilaires peuvent être enlisées dans la membrane péritrophique. La masse sanguine renferme quelques gaines de microfilaires. 


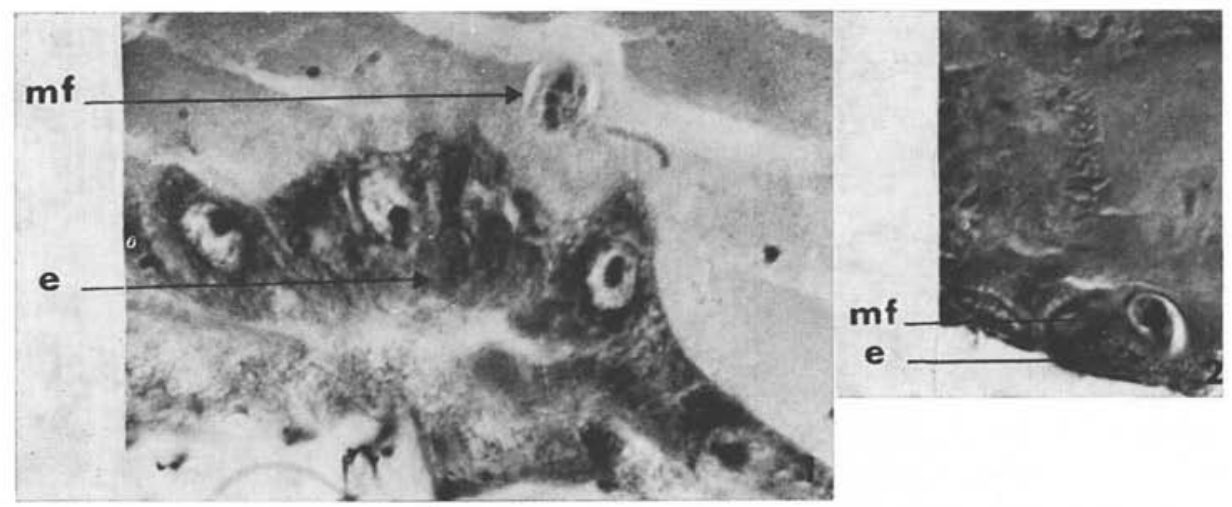

FIG. 2. - Wuchereria bancrofti chez Anopheles gambiae A. - Photo 1: une microfilaire stomacale près d'un groupe de cellules hautes en éventail, $1 \mathrm{~h}$ après le repas; - photo 2: formation épithéliale «en boule» de la figure F ( $3 \mathrm{~h}) ;-$ photo 3 : formation épithéliale "en parasol » de la figure D et E (12 h)

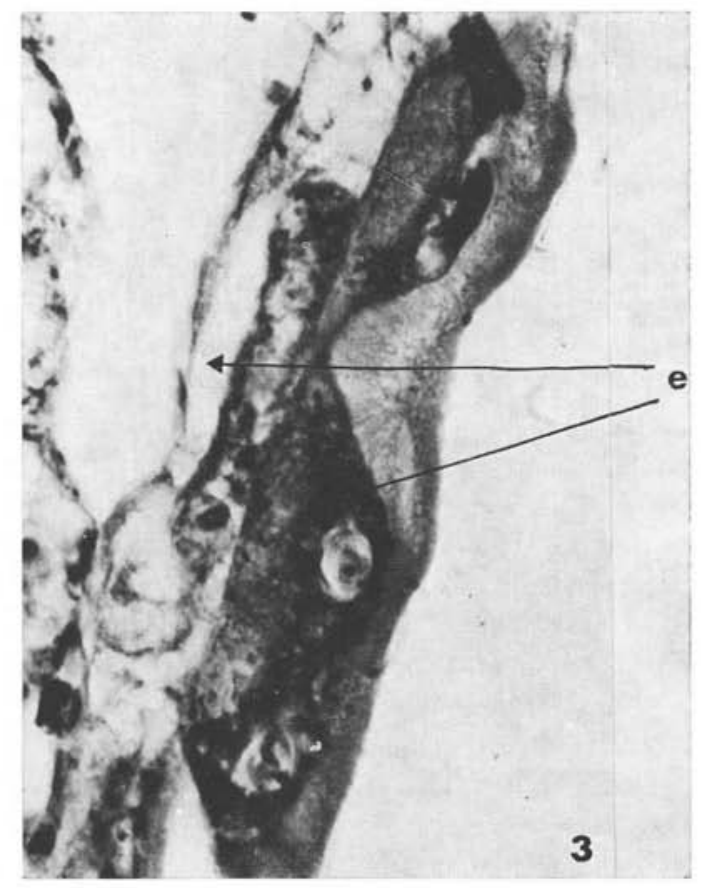

\section{2) Traversée de la paroi stomacale.}

Aucune microfilaire en train de traverser complètement la paroi stomacale n'a été observée. Par contre 6 des 32 étudiées présentent des sections de microfilaires situées dans les cellules épithéliales digestives de l'estomac ; ces + ont été fixées respectivement à 30' (822 JA et $823 \mathrm{JA}), 1 \mathrm{~h}(1363 \mathrm{JA}$ et $1367 \mathrm{JA}), 3 \mathrm{~h}(641 \mathrm{JA})$ et $12 \mathrm{~h}(789 \mathrm{JA})$. Chaque femelle ne renferme qu'une microfilaire intraépithéliale à l'exception de 641 JA qui en a deux. 
Les microfilaires intraépithéliales conservent en fait une partie du corps (la région postérieure) dans le sang et le précurseur de la membrane péritrophique; la région antérieure est lovée dans plusieurs cellules contiguës; la tête de la microfilaire est généralement située à la base des cellules épithéliales et étire parfois la membrane basale.

Les cellules parasitées sont fréquemment ventrales, plus rarement dorsales et sont antérieures ou postérieures. Ce sont des cellules épaisses: soit des cellules pincées à la base, identiques à celles qui existent chez les Anophèles sains (fig. $1 \mathrm{~B}$ et $\mathrm{C}$ ), soit des formations cellulaires très particulières : chez $641 \mathrm{JA}(3 \mathrm{~h})$, une des microfilaires parasite une cellule arrondie, en forme de boule (fig. $1 \mathrm{~F}, \mathrm{pl} .1$, photo 2 ) dont le bord externe est étroitement appliqué à l'épithélium digestif ; chez 789 JA (12 h), l'image est assez semblable mais beaucoup plus volumineuse (fig. 2, photo 3): la microfilaire est située dans une grosse masse cytoplasmique en forme de parasol, à bordure striée, qui est reliée à l'épithélium par un pont cytoplasmique très grêle (fig. $1 \mathrm{D}$ et $\mathrm{E}$ ) ; les bords de cette formation retombent sur l'épithélium avoisinant qui, à ce niveau, est dépourvu de bordure striée.

Le cytoplasme des cellules parasitées montre généralement une forte coloration basophile, parfois localisée (fig. $1 \mathrm{~B}$ et $\mathrm{C}$ ), un aspect tourmenté et des inclusions basophiles ou acidophiles; le passage très récent d'une microfilaire peut laisser une vacuole dans le cytoplasme (fig. 1 B). Certains noyaux au voisinage de la microfilaire prennent un aspect picnotique (fig. $1 \mathrm{~B}, \mathrm{E}$ et $\mathrm{F}$ ).

\section{3) Particularités de l'épithélium stomacal des 9 infestées.}

L'épithélium stomacal des $\subsetneq$ infestées présente de nombreuses formations \& en boule» et en «parasol » qui ne renferment pas de microfilaires, mais qui ont, comme celles décrites au paragraphe précédent, un cytoplasme anormal caractérisé par sa désorganisation, sa basophilie (il est coloré en violet par le Vert de méthyle-Pyronine) et la présence de noyaux picnotiques (colorés en vert par la même coloration). L'ensemble de ces trois caractères se retrouve aussi souvent chez les petits groupes de cellules disposées en éventail.

Les formations en «boule » et en «parasol » ont une base large (fig. $1 \mathrm{G}$ à I) ou réduite à un fin pédoncule (fig. $1 \mathrm{D}$ et E.). Elles renferment souvent une gaine de microfilaire (fig. $1 \mathrm{G}$ et ) ; cette gaine est parfois semi-intra-épithéliale, une partie restant dans la lumière stomacale.

Ces formations sont peu fréquentes à $1 \mathrm{~h}$, au début du passage des microfilaires vers l'hémocèle mais sont nombreuses vers la $6^{\circ} \mathrm{h}$, quand la majorité des microfilaires a atteint l'hémocèle ; à la $6^{\circ} \mathrm{h}$, chez 2 q ayant respectivement 4 et 6 microfilaires passées dans la cavité abdominale et le thorax, ces formations sont moins nombreuses que chez une $3^{\circ} q$ ayant 23 microfilaires passées dans l'hémocèle.

Entre $3 \mathrm{~h}$ et $6 \mathrm{~h}$, l'épithélium qui est au voisinage des zones hypertrophiées est aminci, parfois très localement broyé ou même détruit (fig. $1 \mathrm{G}$ ) ; chez les 9 fixées à $12 \mathrm{~h}$, cet épithélium est continu et épais (fig. $1 \mathrm{D}$ et $\mathrm{E}$ ). A la $21^{\circ} \mathrm{h}$, l'épithélium est régulier et les volumineuses formations basophiles ont disparu. 


\section{Conclusions.}

1) Mode de traversée de la paroi stomacale par les microfilaires.

L'absence d'embrochage de la paroi stomacale et la présence de microfilaires intraépithéliales montrent que les microfilaires quittent l'estomac en pénétrant aisément dans les cellules stomacales mais que la lame basale résistante retarde leur arrivée dans l'hémocèle; la proportion relativement faible des microfilaires intraépithéliales suggère toutefois une traversée assez rapide de la paroi stomacale.

Les microfilaires intraépithéliales sont trop grandes pour se lover entièrement dans les cellules et une partie du corps reste dans la masse sanguine et le coagulum péritrophique; la pénétration dans la cellule se fait par la tête. Les microfilaires pénètrent dans des cellules hautes, dont l'apex renflé est facilement perforé par des microfilaires qui sont plus ou moins parallèles à la paroi stomacale. La situation de ces cellules parasitées est quelconque mais surtout ventrale.

Les microfilaires intraépithéliales ont été observées au niveau de l'estomac et jamais au niveau du cardia. Les microfilaires arrivent done dans les muscles thoraciques après avoir cheminé dans la cavité abdominale.

\section{2) Réactions de l'épithélium digestif au passage des microfilaires.}

Les cellules parasitées ont un cytoplasme localement basophile, désorganisé, et des noyaux picnotiques. Ces caractères se retrouvent dans des formations épithéliales très particulières, absentes chez les $q$ saines, en forme de boule et de parasol, qui contiennent fréquemment des gaines de microfilaires. Ces excroissances sont donc dues au passage des microfilaires; cette conclusion est corroborée par le fait que les hypertrophies épithéliales sont rares quand débute la traversée de la paroi stomacale, vers $1 \mathrm{~h}$, et deviennent nombreuses chez les $\uparrow$ de $6 \mathrm{~h}$, principalement chez celles qui ont beaucoup de microfilaires passées dans l'hémocèle.

Jusqu'à la $6^{\mathrm{e}}$ heure, ces formations reposent sur un épithélium digestif aminci ou même détruit (la membrane basale exceptée) tandis que vers la $12^{\circ}$ heure, l'épithélium sous-jacent est épais; à la $21^{\circ}$ heure les excroissances épithéliales sont absentes. Nous pensons pouvoir expliquer ces faits de la façon suivante: la pénétration d'une microfilaire dans un groupe de cellules provoque une abrasion très localisée de l'épithélium (fig. $1 \mathrm{G}$ ) tandis que le cytoplasme avoisinant réagit par une hypertrophie (fig. $1 \mathrm{G}$ ). L'épithélium détruit va être rapidement régénéré par les cellules épithéliales saines qui n'ont pas été modifiées lors du passage des microfilaires; l'excroissance épithéliale n'intervient pas dans cette régénération, et lorsque l'épithélium digestif est cicatrisé, elle se trouve ainsi appliquée contre un épithélium parfaitement sain (fig. 1 D et E). Cette excroissance qui n'est plus reliée à l'épithélium que par un fin cordon cytoplasmique, doit finalement tomber dans la lumière stomacale.

3) Confrontation des observations histologiques et des résultats numériques.

La membrane péritrophique n'est pas un obstacle pour les microfilaires pendant 1a phase principale de la traversée de la paroi stomacale (6 premières heures); le fait que 
seulement $30 \%$ en moyenne des microfilaires ingérées passent dans l'hémocèle semble dû uniquement à la lyse des microfilaires dans l'estomac.

L'analyse numérique de la traversée de la paroi stomacale a révélé que le nombre des microfilaires passées dans l'hémocèle augmente avec le nombre des microfilaires ingérées ; mais elle montre aussi que cette proportion est variable et s'élève quand le nombre des microfilaires ingérées dépasse une moyenne critique (environ 30 microfilaires). En histologie, nous avons vu que les microfilaires traversent les zones épithéliales épaisses et que leur passage détermine une réaction qui aboutit à la formation d'excroissances épithéliales volumineuses. Ces observations expliquent, à notre avis, le phénomène de facilitation mis en évidence lors des dissections, car les microfilaires engendrent ainsi elles-mêmes de nouveaux sites particulièrement favorables au passage d'autres microfilaires.

\section{II. - WUCHERERIA BANCROFTI - AEDES AEGYPTI}

Le porteur de microfilaires est le même que précédemment.

Les Aedes proviennent de Kongolikan (Haute-Volta); la souche est entretenue à $27^{\circ}$; les $q$ utilisées sont âgées de 6 à 9 jours.

Etude histologique de l'estomac d'Aedes aegypti gorgé sur Homme (ou Veau) sain. Son évolution pendant les premières 24 heures après le repas.

La morphologie de l'estomac est identique quand les Aedes sont gorgés sur Veau ou sur Homme.

Les + ont été fixées à $3 \mathrm{~h}, 12 \mathrm{~h}$, et $24 \mathrm{~h}$.

L'épithélium digestif est distendu et mince $(2 \mu$ à $5 \mu$ d'épaisseur) sauf dans la région préproctodéale où il atteint $10 \mu$ à $25 \mu$ d'épaisseur ; dispersés ça et là, quelques groupes de cellules digestives disposées en éventail ne sont pas étirées et sont plus hautes que l'épithélium environnant (10 $\mu$ à $30 \mu$ de haut) ; quand l'épithélium digestif est très étiré il prend une coloration bleu-vert (hémalun-éosine). A la $24^{\circ}$ heure, l'épithélium est épaissi ; le cytoplasme cellulaire est dense; les noyaux sont vésiculeux avec un gros nucléole. La bordure striée est surtout visible dans les zones les moins distendues.

A la $3^{\circ}$ heure, le repas de sang est entouré par un coagulum dense, bien délimité et peu épais $(3 \mu$ à $15 \mu)$. Plus tard $\left(12^{\circ}\right.$ heure $)$, une membrane péritrophique de structure lamelleuse apparaît à l'extérieur ou à l'emplacement du coagulum (fig. 3, photo 1 et 2) ; elle est mince $(1 \mu$ à $3 \mu)$, régulière et formée par 2 ou 3 lamelles. A la $24^{\circ}$ heure le repas de sang est enfermé dans une croûte brunâtre de $5 \mu$ à $15 \mu$ d'épaisseur dont la structure est hétérogène : elle est grenue vers le centre et lamelleuse à la périphérie ; le nombre des lamelles est sensiblement le même qu'à $12 \mathrm{~h}$.

Etude histologique de la traversée de la paroi stomacale par les microfilaires.

$19 q$ ont été fixées et observées : $2 q$ à $30,3 q$ à $1 \mathrm{~h}, 3$ à $3 \mathrm{~h}, 3 q$ à 6 h et $7 q$ à $12 \mathrm{~h}$. 

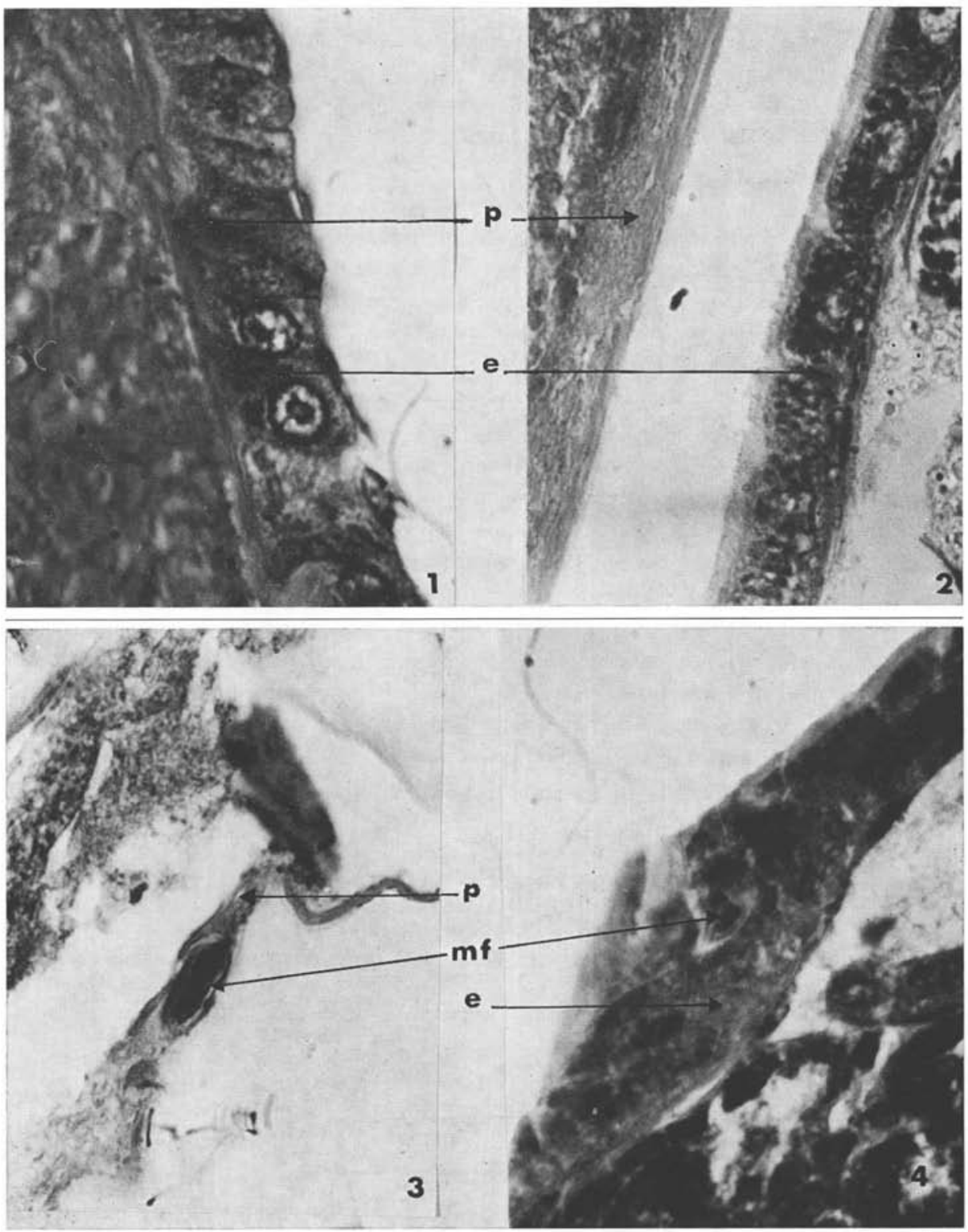

FIg. 3. - Wlichereria bancrofti chez Aedes aegypti. - Photo $1:$ membrane péritrophique à $12 \mathrm{~h}$; - photo 2: idem, autre aspect; - photo $3:$ une microfilaire enlisée dans une lamelle péritrophique à $12 \mathrm{~h} ;-$ photo 4 : une section de microfilaire intraépithéliale à $6 \mathrm{~h}$ 


\section{1) Répartition des microfilaires dans le repas de sang.}

Les microfilaires se répartissent dans toute la masse sanguine. Jusqu'à la $6^{\circ}$ heure, elles traversent aisément le coagulum péritrophique ; à la $12^{\mathrm{e}}$ heure, les microfilaires sont bloquées par les minces lamelles péritrophiques (fig. 3, photo 3). La membrane péritrophique des $q$ infestées est analogue à celle des $q$ saines, ni plus mince, ni plus épaisse.

\section{2) Traversée de la paroi stomacale.}

Sur les 19 fixées, 8 ont des sections de microfilaires intraépithéliales (fig. 3, photo 4 et fig $4 \mathrm{~A}): 1$ sur 3 à $1 \mathrm{~h}$ ( 1 microfilaire), 3 sur 3 à $3 \mathrm{~h}$ ( 3 microfilaires chez 1079 JA ; 2 microfilaires chez $1037 \mathrm{JA} ; 1$ microfilaire chez $1036 \mathrm{JA}), 3 q$ sur 3 à $6 \mathrm{~h}$ (3 microfilaires chez 399 JA ; 5 microfilaires chez 397 JA ; 11 microfilaires chez 396 JA), 1 \& sur 7 à $12 \mathrm{~h}$ (1 microfilaire chez $440 \mathrm{JA})$. Au total 27 microfilaires intraépithéliales ont été observées, les $q$ les plus infestées étant celles fixées à la $6^{\circ}$ heure.

Ces microfilaires ne sont jamais entièrement situées dans les cellules digestives; une partie du corps (la région postérieure) reste dans le sang et le coagulum péritrophique (fig. 4, photo 2 ) ; plusieurs microfilaires ont la région antérieure passée dans l'hémocèle $(6$ microfilaires sur 27 réparties chez les $q$ suivantes : 1 à $1 \mathrm{~h}, 2$ à $3 \mathrm{~h}, 3$ à $6 \mathrm{~h}$ et 1 à $12 \mathrm{~h}$ ). On voit aussi des microfilaires pénétrer dans l'épithélium digestif en découpant en biais un fragment de cellules qui se trouve ainsi en partie détaché de l'épithélium (fig. 4, photo 3).

Les microfilaires intraépithéliales se rencontrent aussi bien dans la région antérieure que dans la région postérieure de l'estomac, et ventralement que dorsalement; toutefois chez le Moustique le plus riche (396 JA), la majorité des microfilaires intraépithéliales est située dans la région postérieure.

Les microfilaires parasitent assez souvent les groupes de cellules épaisses disposées en éventail (10 cas sur 27).

\section{3) Réaction de l'épithélium au passage des microfilaires.}

Il n'y a pas d'hypertrophie cellulaire importante mais seulement une réaction localisée du cytoplasme qui devient basophile au contact de la microfilaire ; certains noyaux sont picnotiques.

\section{Conclusion.}

Dans le couple $W$. bancrofti - A. aegypti, le nombre des microfilaires intraépithéliales est particulièrement élevé ; ce nombre augmente en fonction du temps, atteint sa plus haute valeur à la $6^{\circ}$ heure et diminue ensuite.

Ces données histologiques, confrontées avec les résultats numériques (arrivée des microfilaires dans l'hémocèle de $1 \mathrm{~h}$ à $24 \mathrm{~h}$ ) permettent d'exposer le mécanisme de la traversée de la paroi stomacale d' $A$. aegypti par les microfilaires de $W$. bancrofti de la façon suivante: un faible pourcentage de microfilaires atteint assez vite l'hémocèle (1 microfilaire en moyenne à $2 \mathrm{~h}$ ) tandis que la plupart d'entre elles pénètrent tardivement 
dans les cellules digestives : entre 3 et $6 \mathrm{~h}$, mais avant que la membrane péritrophique ne devienne un barrage efficace $\left(12^{\circ}\right.$ heure). Le fait que les arrivées des microfilaires dans l'hémocèle soient en nombre important jusqu'à $24 \mathrm{~h}$, montre que les microfilaires séjour-

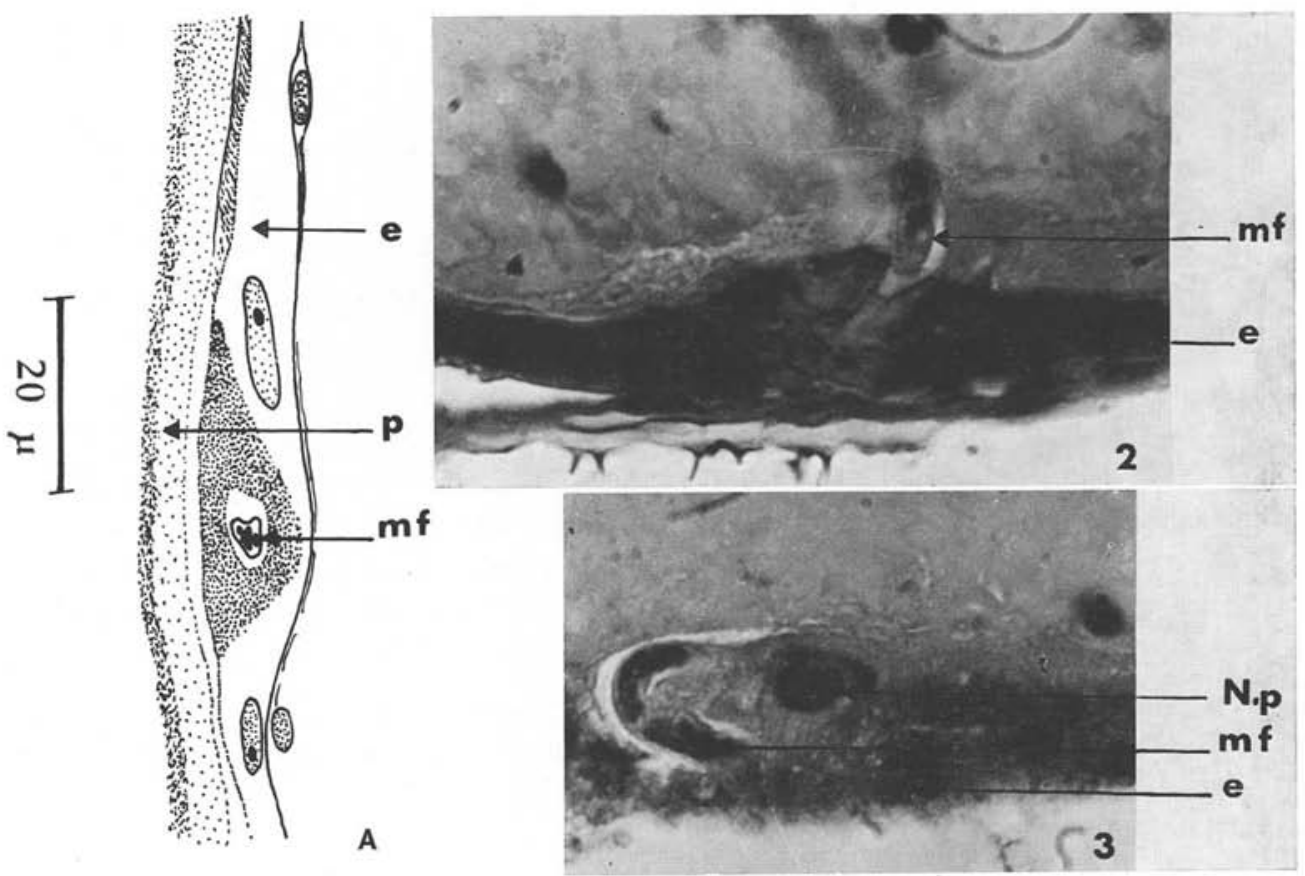

FIG. 4. - Wuchereria bancrofti chez Aedes aegypti. - A: une microfilaire intraepithéliale, à la $6^{e} \mathrm{~h}$; - Photo 2: une microfilaire qui pénètre dans un groupe de cellules épaisses; - Photo 3 : une microfilaire pénètre en biais dans l'épithélium; la cellule fendue est grosse avec un volumineux noyau picnotique

Symboles :

$\mathrm{e}=$ épithélium.

$p=$ membrane péritrophique.

$m f=$ microfilaire

$s=$ sang.

$g$ = gaine.

$\mathrm{N} p=$ noyau picnotique.

nent longuement dans l'épithélium digestif (jusqu'à $12 \mathrm{~h}$ environ); la membrane basale d'A. aegypti est un obstacle particulièrement difficile à franchir pour les microfilaires de W. bancrofti.

Aucune image histologique ne permet, par contre, d'expliquer la limitation des passages des microfilaires dans l'hémocèle, mise en évidence au cours des dissections : aucune saturation de l'épithélium digestif par les microfilaires n'a été observée, ni d'épaississement de la membrane péritrophique chez les $q$ les plus riches en microfilaires stomacales. 


\section{III. - SETARIA LABIATOPAPILLOSA - AEDES AEGYPTI}

Le porteur de microfilaires est un Veau infesté expérimentalement. Les microfilaires mesurent de $240 \mu$ à $310 \mu$ de long sur $6 \mu$ à $7,5 \mu$ de large ; elles se développent dans les muscles thoraciques du Moustique.

Etude histologique de la traversée de la paroi stomacale par les microfilaires.

10 moustiques ont été fixés et observés : 1 à $1 \mathrm{~h}, 3 q$ à $2 \mathrm{~h}, 2$ à $3 \mathrm{~h}$ et 4 à $6 \mathrm{~h}$.

Les microfilaires sont ingérées en nombre trop réduit pour pouvoir étudier leur répartition dans l'estomac.

1) Traversée de la paroi stomacale.

4 o ont des microfilaires intraépithéliales; elles ont été fixées respectivement à $1 \mathrm{~h}$ ( 1 \& avec 1 microfilaire intraépithéliale), à $2 \mathrm{~h}(2$ \& avec chacune 1 microfilaire intraépithéliale), à $3 \mathrm{~h}$ (1 ㅇ avec 1 microfilaire intraépithéliale).

Ces microfilaires sont situées dans des plis de l'épithélium digestif, aux cellules particulièrement épaisses. Une partie du corps reste dans la lumière de l'estomac. Certaines microfilaires sont en train de passer dans l'hémocèle.

2) Réaction de l'épithélium digestif au passage des microfilaires.

Les cellules réagissent au passage des microfilaires: le cytoplasme devient basophile, les noyaux proches du parasite sont picnotiques.

\section{Conclusion.}

Les relations numériques entre microfilaires ingérées et microfilaires passées dans l'hémocèle sont analogues chez $W$. bancrofti - A. aegypti et Setaria labiatopapillosa A. aegypti; dans les deux cas il existe un phénomène de limitation que l'étude histologique ne permet pas d'expliquer. Mais le mode de passage des microfilaires de S. labiatopapillosa diffère de celui de $W$. bancrofti par sa grande rapidité, la membrane basale étant dans ce cas aisément traversée par les microfilaires (rareté des microfilaires intraépithéliales, et arrivée des microfilaires dans l'hémocèle pendant les 6 premières heures qui suivent le repas).

\section{IV. - CONCLUSIONS}

Quelques faits ressortent de l'analyse histologique de ces 3 couples filaires - vecteurs :

1) Quelle que soit la filaire et le vecteur, les microfilaires quittent le tube digestif au niveau de l'estomac et migrent ensuite de l'abdomen vers les muscles thoraciques; 
ces résultats sont conformes à ceux de Wharton (1957) obtenus sur le couple Brugia malayi - Mansonia longipalpis; les passages directs du cardia vers l'hémocèle, signalés par O'Connor et Beatty (1936) et par Iyengar (1936) dans le couple W. bancrofti Culex fatigans, n'ont pas été observés.

2) La traversée de l'estomac par les microfilaires comporte une phase intraépithéliale ; les microfilaires, qui pénètrent aisément dans les cellules épithéliales gonflées (souvent localisées dans la région postérieure de l'estomac), sont bloquées par la lame basale péristomacale ; ce blocage est particulièrement net chez $W$. bancrofti - A. aegypti : la longue durée du séjour intraépithélial explique l'arrivée très tardive (jusqu'à 24 heures) des microfilaires dans l'estomac, alors que le barrage périthrophique (installé dès la $12^{\circ}$ heure) ne permet plus aux microfilaires de pénétrer dans les cellules digestives : un phénomène analogue, mais encore plus marqué, a déjà été observé chez Onchocerca volvulus : Simulium damnosum (Bain et Philippon, 1970).

Le séjour intraépithélial des microfilaires dans la paroi stomacale du vecteur, observé chez de nombreux couples filaires - vecteurs (Esslinger, 1962 ; Bain et Philippon, 1970), paraît être un phénomène très général, probablement vestige des localisations intracellulaires des embryons d'ancêtres Spirurides.

3) L'analyse numérique de la traversée de la paroi stomacale des moustiques par les microfilaires fait ressortir deux types de relations quantitatives entre les microfilaires passées dans l'hémocèle : un phénomène de facilitation chez $A$. gambiae $\mathrm{A}$ et un phénomène de limitation chez $A$. aegypti (Brengues et Bain, sous presse). L'étude histologique n'a pas permis de comprendre la limitation ; par contre, la paroi stomacale d' $A$. gambiae A présente des images très particulières qui semblent expliquer la facilitation : chez ce moustique, l'épithélium digestif réagit fortement au passage des microfilaires en formant de volumineuses excroissances ; ces zones épithéliales épaisses et saillantes constituent de nouveaux sites tout à fait favorables à la pénétration des microfilaires; ainsi les passages sont d'autant plus nombreux que l'épithélium est attaqué par un nombre plus important de microfilaires.

\section{Bibliographie}

Bain (O.) et Philippon (B.), 1970. - Mécanisme de la traversée de la paroi stomacale par les microfilaires chez Anopheles stephensi et Simulium damnosum. Mise en évidence d'un séjour des microfilaires dans l'épithélium digestif. Ann. Parasit., 45, 295-320.

Brengues (J.) et Bain (O.) (sous presse). - Epidémiologie des Filarioses transmises par les moustiques, en Afrique Occidentale. I. Passage des microfilaires de Wuchereria bancrofti dans l'hémocèle d'Anopheles gambiae et d'Aedes aegypti et des microfilaires de Setaria labiatopapillosa dans l'hémocèle d'Ae. aegypti.

EssLinger (J. H.), 1962. - Behaviour of microfilaria of Brugia pahangi in Anopheles quadrimaculatus. Amer. J. trop. Med. Hyg., 11, 749-758. 
IYengAR (M. O. T.), 1936. - Entry of Filaria larvae into the body cavity of the mosquito. Parasitology, 28, 190-194.

O'ConNor (F. W.) et BeATtY (H.), 1936. - The early mignations of Wuchereria bancrofti in Culex fatigans. Trans. Roy. Soc. Trop. Med. Hyg., 30, 125.

WharTon (R. H.), 1957. - Studies on filariasis in Malaya : observations on the development of Wuchereria malayi in Mansonia (Mansonioides) longipalpis Ann. trop. Med. Parasit., 51, 278-296. 